\title{
Brain Tumor Segmentation in MRI Images
}

\author{
Adarsh Dhiman, Prof. B.S. Satpute
}

\begin{abstract}
It is very hard to detect brain tumor in the beginning as the human brain has an extremely intricate structure. This is a very cumbersome process for the radiologist to detect the tumor, physically segment and recognize the tumor in the MRI image. The machine learning is ideal for this task as it is great at perceiving tumor locales and there is no need of undertaking specific algorithm. Before the advent of the deep learning applications, a specialist in the field of medical domain used to construct the features and it demanded the special knowledge in the medical field. To eliminate the domain-specific knowledge; we are using the U-Net based model to carry out the brain tumor segmentation. The U-Net network, which is based on deep learning, is capable of extracting the hierarchical features present in MRI images without any manual intervention. The idea is to use this domain-independent algorithm to carry out the segmentation task automatically. In this paper, we recommend using U-Net based deep convolutional neural network to carry out brain tumor segmentation. U-Net based Convolutional Neural Network is expected to improve segmentation accuracy. We have used BRATS 2017 dataset, to train the neural network and henceforth carry out brain tumor segmentation.
\end{abstract}

Index Terms - Deep Learning, Machine Learning, Brain Tumor, Convolutional Neural Network.

\section{INTRODUCTION}

Medical imaging techniques like MRI had made tremendous progress and it had made it a reality to diagnose the disease at earlier stages. The radiologist manually examines these scans to interpret the presence of tumor in the MRI. It is a very cumbersome errand to interpret such an immense volume of scans at a stretch and daily ongoing process. There is a huge possibility of error in this manual process. There is a need to automate this process [3]. There are a plethora of organizations which are making this kind of data available for research. We are utilizing BRATS 2017 dataset to train our neural network and segment the MRI image thereafter training. This BRATS 2017 dataset has multimodal images like low grade and high-grade tumor MRI scans. It is next to impossible to interpret this much volume of information. Machine learning techniques are ideal to mine this volume data to interpret and obtain results automatically.

Manuscript revised on September 23, 2019 and published on October 10,2019

Adarsh Dhiman, Dept. of Computer Engineering, DPU, Pune, India.

Prof. B.S. Satpute, Dept. of Computer Engineering, DPU, Pune, India.
The U-Net model is perfect to separate salient features from data, which will assist the radiologist with making better choices. U-Net model extracts various leveled data features from MRI images to create a model that provides insight into the data. The features extracted from the data are automated and they provide meaningful deeper insights, which are not even obvious to naked eye. These various leveled features are self-learned by the U-Net based convolutional neural network model [1].

\section{LITERATURE REVIEW}

A. U-Net based deep Convolutional Neural Networks for Brain Tumor Segmentation [1]

In this paper [1], we become more acquainted with how the U-Net based Convolutional Neural Network handles the variable edge problem if there should be an occurrence in MRI picture (cerebrum tumor).

The skip architecture is intended to manage variable edge issue. This architecture has achieved promising outcomes on MRI pictures. In this investigation, the authors have utilized U-Net based Convolutional Neural Network as a base model. This design has indicated great outcomes in tumor segmentation.

\section{B. Stacked De-noising Auto-Encoder based Deep Learning Framework [2]}

In this paper [2], deep learning is introduced to automatically segment brain tumor and avoid the manual segmentation and domain knowledge. This paper introduces the concept of extracting image patches from MRI image and then provides the gray level image patches as input to Stacked De-noising Auto-Encoder. In order to carry out feature classification the Stacked De-noising Auto-Encoder (SDAE), automatically learns from the data in the MRI image. In this study, the result is mapped on binary image and eventually the final segmentation is achieved.

\section{Deep Learning for MRI Image Analysis [3]}

In this paper [3], author discussed different deep learning neural networks like Feed-Forward Neural Networks, Convolutional Neural Networks etc. and explained their application in MRI Image Analysis for image segmentation and detection of tumor.

\section{Image Segmentation methods and Convolutional Neural Network [4]}

In this paper [4] author discusses, the different methods which handles semantic division such as pixel-wise division. 
In this case a small patch of picture is used to order the middle pixel, and the fully convolutional designs where the system is provided with whole picture and the output is a semantic division volume. This study used Convolutional Neural Network for MRI image segmentation.

\section{E. Brain Tumor Segmentation with aid of Convolutional Neural Network [5]}

In this paper [5], author used Convolutional Neural Network for brain tumor segmentation in medical image. In this study the preprocessing step consists of bias correction and patch normalization. In this study the existing low-grade glioma (LGG) samples are rotated to produce new LGG samples. The deep Convolutional Neural Network is built using $3 \times 3$ kernels on top of convolutional layers.

\section{MACHINE LEARNING FOR BRAIN TUMOR SEGMENTATION}

The images are manually labelled by domain expert to train the neural network. The idea is to use the fully Convolutional Neural Network based on deep learning which will be trained on the input data and will learn the features present in MRI image without any manual intervention. The model generated in due course will be used to segment the MRI image and detect the necrotic and enhancing tumor.

\section{A. Existing System and Disadvantages}

Traditionally the features were hand crafted by the domain specialist. This process required the domain specific knowledge. These images were then used to train the neural network. This process consumes a lot of time and labor intensive. The loss function used in the current system is not apt for the problem in hand. The following are the disadvantages of established system:

- Error Prone

- Time Consuming

- Manual

- Requires Domain Knowledge

- Human Fatigue

\section{B. Proposed System and Advantages}

In proposed system we used deep learning techniques based on U-Net architecture. The system performs computer aided segmentation and detection of tumor. The success of the proposed system comes from the following factors:

- Use of high end CPUs and GPUs

- Discovery of Hierarchical Features

- Development of Learning Algorithms
The system architecture consists of the following components:

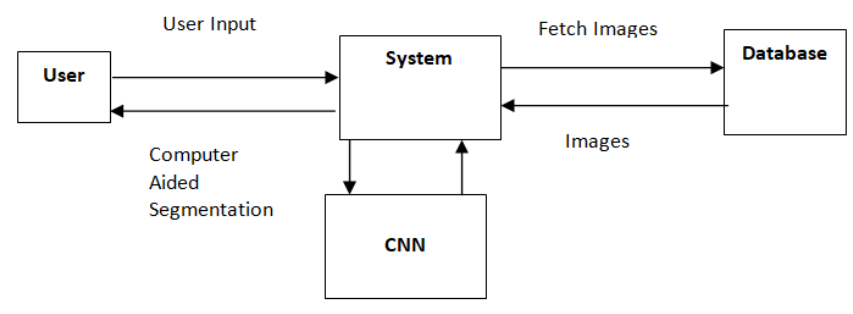

Fig. 1. System Architecture Diagram

\section{b. U-Net Architecture Diagram}

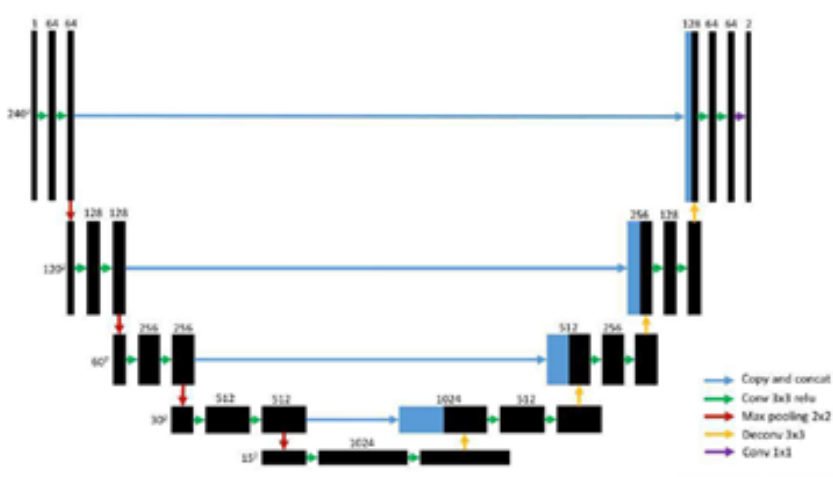

Fig. 2. U-Net Convolutional Neural Network Architecture

In this study the U-Net based network architecture is used, which consists of a down-sampling path and an up-sampling path as indicated in "Fig. 2". The contraction path or down-sampling consists of four blocks. In this scheme every block has $3 \mathrm{X} 3$ convolutional layers and ReLU activation. The down sampling or contraction path consists of a $2 \times 2$ Max Pool which is added to the end of every block, to reduce feature map. The expansion path or up-sampling path has also four blocks. All the blocks in the expansion or up-sampling path consists of a de-convolutional layer, a link to the corresponding feature map in the down-sampling path and $3 \mathrm{X} 3$ convolutional layers and ReLU activation.

\section{c. Segmentation Algorithm}

The algorithm used in U-Net based Convolutional Neural Network is explained below in "Fig. 3". This architecture has a contraction path and an expansion path. 


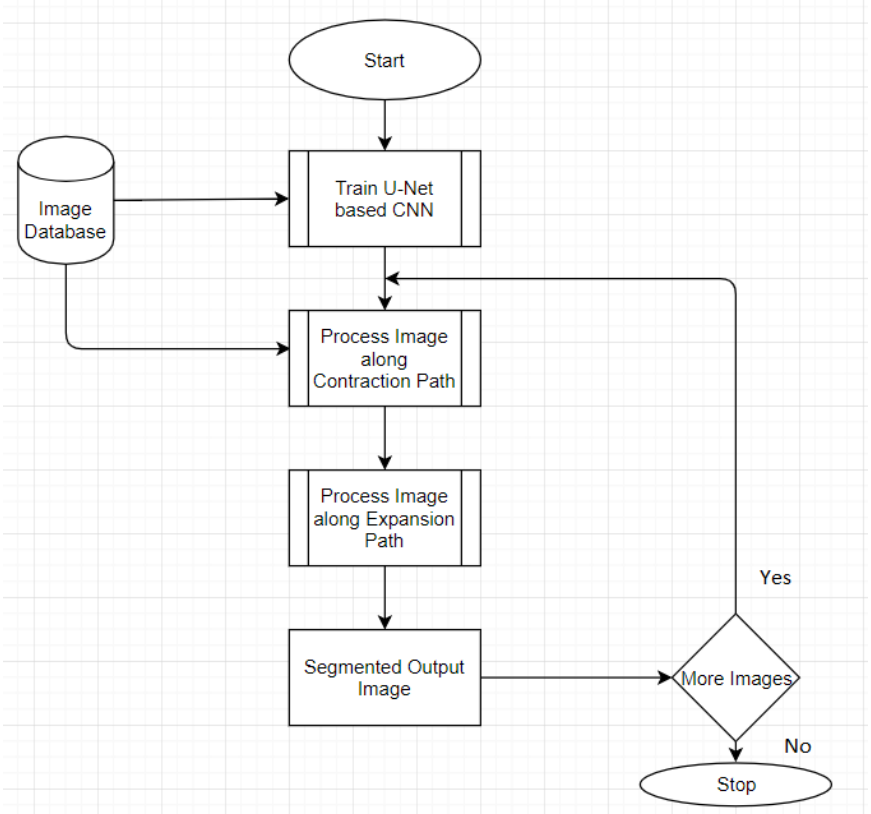

Fig. 3. Algorithm

\section{d. Method to verify and assess results}

The quality of segmentation result of the U-Net based Convolutional Neural Network "Fig. 4" is calculated based on the dice similarity coefficient which is the ratio between the manually labeled brain tumors by radiologist and the segmentation results obtained from the deep convolutional neural network. The formula for dice similarity coefficient and sensitivity are evaluated as explained below:

$$
\begin{aligned}
& \mathrm{DSC}=\frac{2 \mathrm{TP}}{\mathrm{FP}+2 \mathrm{TP}+\mathrm{FN}} \\
& \text { Sensitivity }=\frac{\mathrm{TP}}{\mathrm{TP}+\mathrm{FN}} .
\end{aligned}
$$

\section{Fig. 4. Evaluation Function}

In the formula mentioned above, the abbreviations are as below:

TP means True Positive.

FP means False Positive.

FN means False Negative.

\section{IMPLEMENTATION DETAILS}

We have used BRATS 2017 dataset to train the model. The dataset has both high grade glioma as well as low grade glioma cases. The dataset has multimodal MRI images which are T1-weighted, T2-weighted and FLAIR which is present for each patient. The dataset is divided into two halves. The first half is used to train the model and the second half is used for validation. The model is trained to extract necrotic and enhancing tumor. The Anaconda Prompt is used to setup the environment and used to train the model. The system generated two models for necrotic and enhancing tumor individually. In order to train the model it is must to have GPU. The hardware and software requirements of the system are defined as below.

Hardware Requirements:

- $\quad$ System: Intel ${ }^{\circledR}$ Xenon R E3-1545-M v5 2.90 GHz

- Hard Disk: 40 GB

- Monitor: 17 VGA Color

- $\quad$ RAM: 16 GB or above

- GPU: GTX 1080

Software Requirements:

- Programming Language: Python

- TensorFlow

- TensorLayer

- OS: Win $7 / 8 / 10$

- IDE: Anaconda Prompt

\section{A. Data Flow Diagram}

The bird eye view of the functionality performed by the system is represented in following Data flow diagram.

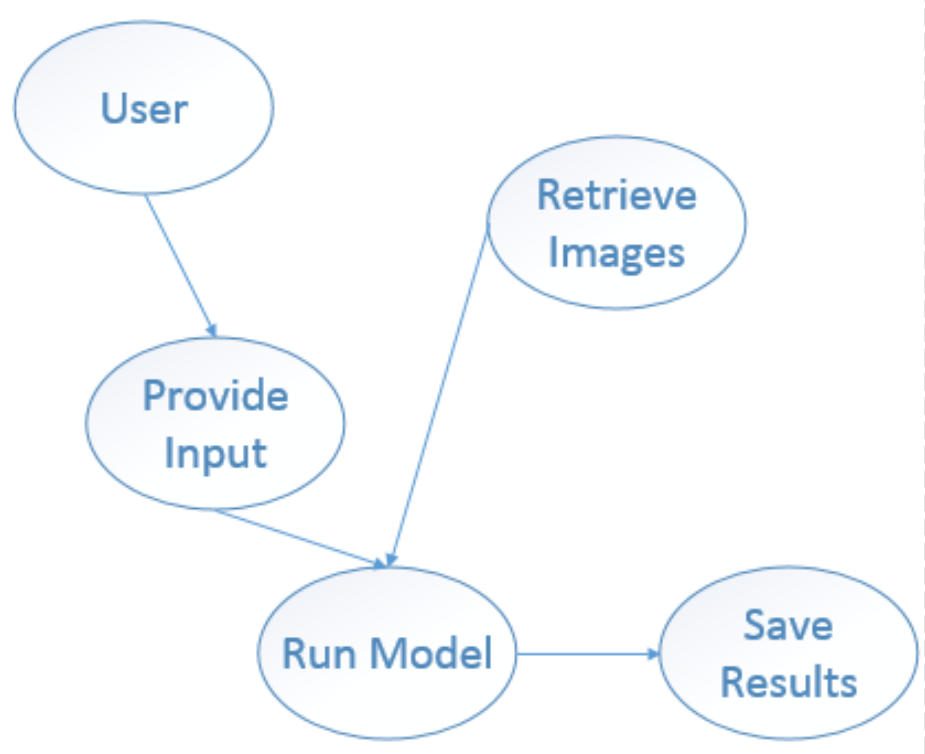

Fig 5: Level 0 Data Flow Diagram

\section{B. Use Case Diagram}

The system and user interaction is explained in the following diagram: 
International Journal of Research in Advent Technology, Vol.7, No.9, September 2019

E-ISSN: 2321-9637

Available online at www.ijrat.org

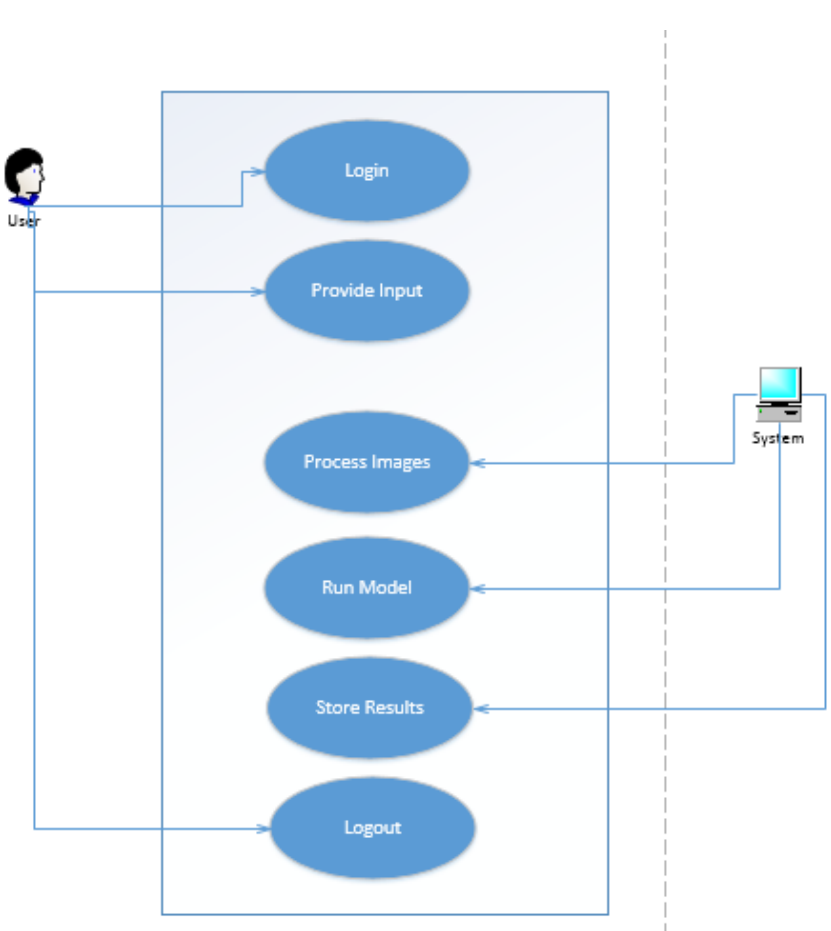

Fig 6: Use Case Diagram

\section{Result And Discussion}

In this study, we used U-Net based fully convolutional neural network for brain MRI image segmentation. Deep learning or deep convolutional neural networks are giving promising results in medical image segmentation based on Dice Similarity Coefficient as compared to other techniques.

The input to the network is a MRI image "Fig. 7", and output is "Fig. 8", the segmented image.

Input Image:

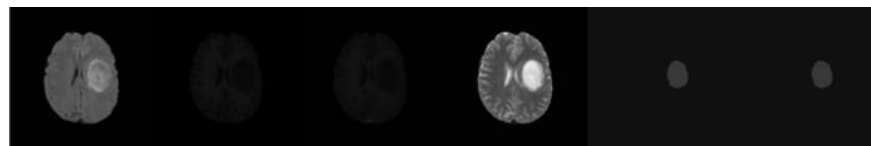

Fig 7: Input MRI Image

Segmented Image:

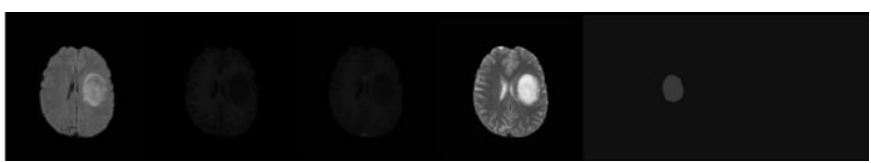

Fig 8: Segmented MRI Image

The chart in "Fig. 9" shows the dice similarity coefficient (ranges between 0 and 1) of the base method and the new proposed method in case of tumor demarcation. The dice similarity coefficient of the proposed method has improved by $5 \%$.

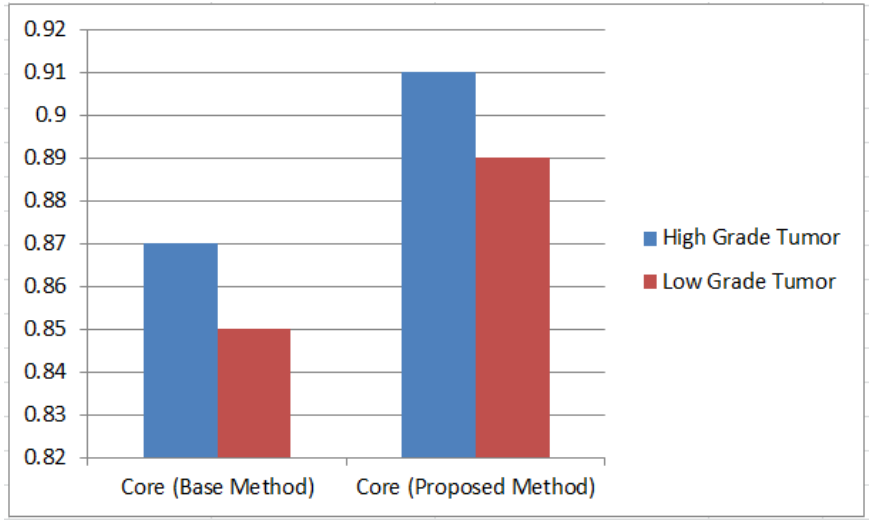

Fig 9: Dice Similarity Coefficient

This network can further be used to study other modalities available in the MRI images to prove the validity and range of network to segment brain MRI images.

\section{Conclusion And Future Scope}

This paper prescribes to utilize Convolutional Neural Network based deep learning methods to segment the MRI image. The progress in image segmentation methods will incredibly aid in automated identification of tumor. The ongoing improvement in deep learning has revealed the cellular features in the MRI images. Deep learning strategies have indicated promising outcomes over various ailments like Alzheimer, Pneumonia and so on; be that as it may, there is still scope for development.

\section{REFERENCES}

[1] Hao Dong, Guang Yang, Fangde Liu, Yuanhan Mo, Yike Guo, "Automatic Brain Tumor Detection and Segmentation Using U-Net Based Fully Convolutional Networks", arXiv:1705.03820 [cs.CV], 3 June 2017.

[2] Zhe Xiao, Ruohan Huang, Yi Ding, Tian Lan, RongFeng Dong and Zhiguang Qin, “A Deep Learning-Based Segmentation Method for Brain Tumor in MR Images", presented at 2016 IEEE 6th International Conference on Computational Advances in Bio and Medical Sciences (ICCABS), Atlanta, GA, USA, Oct. 13-15, 2016.

[3] Shen, Guorong Wu and Heung-Il Suk, "Deep Learning in Medical Image Analysis", Annual Review of Biomedical Engineering, vol. 19, pp. 221-248, June 2017.

[4] Manda SSSNMSRL Pavan, P. Jagadeesh, "Brain Tumor Segmentation using Convolutional Neural Network in MRI Images", International Journal of Pure and Applied Mathematics, vol. 119 no. 17, pp. 1585-1592, 2018.

[5] Sérgio Pereira, Adriano Pinto, Victor Alves, and Carlos A. Silva, "Brain Tumor Segmentation Using Convolutional Neural Networks in MRI Images", IEEE Transactions on Medical Imaging, vol. 35, no. 5, pp. 1240-1251, May 2016.

[6] Hapsari Peni Agustin Tjahyaningtijas, "Brain Tumor Image Segmentation in MRI Image", 2018 IOP Conf. Ser.: Mater. Sci. Eng. 336 012012, vol. 336, conference 1 .

[7] Adnan Qayyuma, Syed Muhammad Anwar, Muhammad Majid, Muhammad Awais, Majdi Alnowamid, "Medical Image Analysis using Convolutional Neural Networks: A Review", Springer US, vol. 42, pp. 226, Oct. 2018.

[8] Kavitha Angamuthu Rajasekaran, Chellamuthu Chinna Gounder, "Advanced Brain Tumor Segmentation from MRI Images", High-Resolution Neuroimaging - Basic Physical Principles and Clinical Applications, ch. 6, pp. 735, 2018.

[9] Adarsh Dhiman, B.S. Satpute, "Brain Tumor Segmentation in MRI Images", International Journal of Engineering and Techniques, vol. 5 Issue 1, pp. 112, Jan-Feb 2019. 
International Journal of Research in Advent Technology, Vol.7, No.9, September 2019

E-ISSN: 2321-9637

Available online at www.ijrat.org

[10] Adarsh Dhiman and B.S. Satpute, "Brain Tumor Segmentation in MRI Images", Eighth Post Graduate Conference of Computer Engineering, 2018-19.

\section{AUTHORS PROFILE}

Adarsh Dhiman, M.E., Dept. of Computer Engineering, DPU, Pune.

Prof. B.S. Satpute, Associate Professor, Dept. of Computer Engineering, DPU, Pune. 